\title{
Network Coding-based Content Distribution in Cellular Access Networks
}

\author{
Claudio Fiandrino, Dzmitry Kliazovich, Pascal Bouvry \\ University of Luxembourg, Luxembourg \\ \{name.surname\}@uni.lu
}

\author{
Albert Zomaya \\ University of Sydney, Australia \\ albert.zomaya@sydney.edu.au
}

\begin{abstract}
Mobile cloud applications have become extremely popular in the last years. Location-based services, navigation, online gaming and social networking are a representative set of "always on" cloud applications in which the same or partially overlapping content is delivered to multiple users. Network coding is a well matching solution to improve content delivery. In this paper we propose the vNC-CELL technique, which uses network coding to combine information flows carrying the same or overlapping content that has to be delivered to co-located users. vNC-CELL executes coding functionalities in a mobile cloud through virtualization as these operations are computationally intensive if performed locally at the base station. Performance evaluation obtained from NS-3 simulations confirms vNC-CELL ability to improve network throughput and reduce download times for the users.
\end{abstract}

Index Terms-Network coding, cellular networks, content distribution.

\section{INTRODUCTION}

Mobile cloud applications are becoming increasingly popular. Ubiquity and mobility are the two main characteristics that attract over 7 billion users [1] in using cloud applications from mobile devices such as smartphones and wearable devices. Mobile devices have become essential for our daily activities as they help to do business, communicate and entertain [2], [3]. As a result, the mobile cloud application market is one of the fastest growing markets and is projected to worth over $\$ 46.90$ billion by 2019 [4]. Mobile data traffic is growing at an unprecedented rate and is projected to generate up to 24.3 EB per month by 2019 [5], which is certainly above the limit of current cellular networks. According to Cisco, nearly $90 \%$ of this mobile traffic will be generated by mobile cloud applications [5]. Addressing the increasing traffic demand is therefore crucial and several solutions have been investigated. Currently, the most adopted techniques either increase network capacity deploying base stations denser and using Device-toDevice (D2D) communications [6] or relieve cellular networks load by performing offloading [7], [8], [9].

Mobile cloud applications, such as navigation, location-based services, online games and multimedia streaming, often deliver the same or partially overlapping content to multiple users. Many of these applications run autonomously in background without requiring explicit inputs from the users. Google Now, for example, anticipates user needs by displaying series of cards created based on the information about the user daily routine, location patterns, information about nearest businesses and restaurants, weather forecasts, road traffic conditions, buses and trains, and other location-based services. For geographically colocated users a lot of this information is likely to be similar. In addition to content similarity, mobile advertisement traffic has to be considered. The majority of the software in applications stores, such as Google Play and Apple Store, is free [10], while revenue comes from in-app advertisement. Being typically composed of static images and text, advertisement files are usually downloaded periodically and account for $7.6 \%$ of the daily user traffic in iPhone devices [11]. When user terminals ask for the same or partially overlapping content, mobile network accomplishes these requests separately and, consequently, uses a large amount of available resources. When these requests come from devices in geographical proximity, they are likely to be served by the same base station. Combining such data flows at the base station has great potential to save network resources [12] and network coding is a well-matching solution for this.

Network coding was originally proposed to improve throughput of multicast communications allowing intermediate node to combine two or more in transit packet together [13], [14]. Throughput enhancement is just one of several possible applications of network coding. Robustness, network tomography and security are other representative examples [15]. For content distribution, network coding is commonly used in peer-topeer networks (P2P). Files are divided into small pieces, also called chunks, and each peer is responsible of content distribution. In this context, network coding improves file download time and augments robustness of the system against user departures that can prevent other peers from retrieving rare chunks [16]. In wireless and cellular networks, network coding is widely adopted to improve throughput and robustness in addition to content distribution [17]. These objectives can be accomplished mitigating packet loss by enforcing error correction techniques like HARQ [18]. In cellular networks, the scenarios typically consider radio node relays, meaning that base station transmissions are relayed at least once before they can reach end users [19], [20], [21], [6]. Although relay nodes are excellent to perform network coding, they are not very common in current cellular networks.

Our previous work [12] proposes a novel network coding based technique, called NC-CELL (Network Coding in CELLular networks) to improve efficiency of content distribution for cloud applications in mobile cellular networks to geographically 


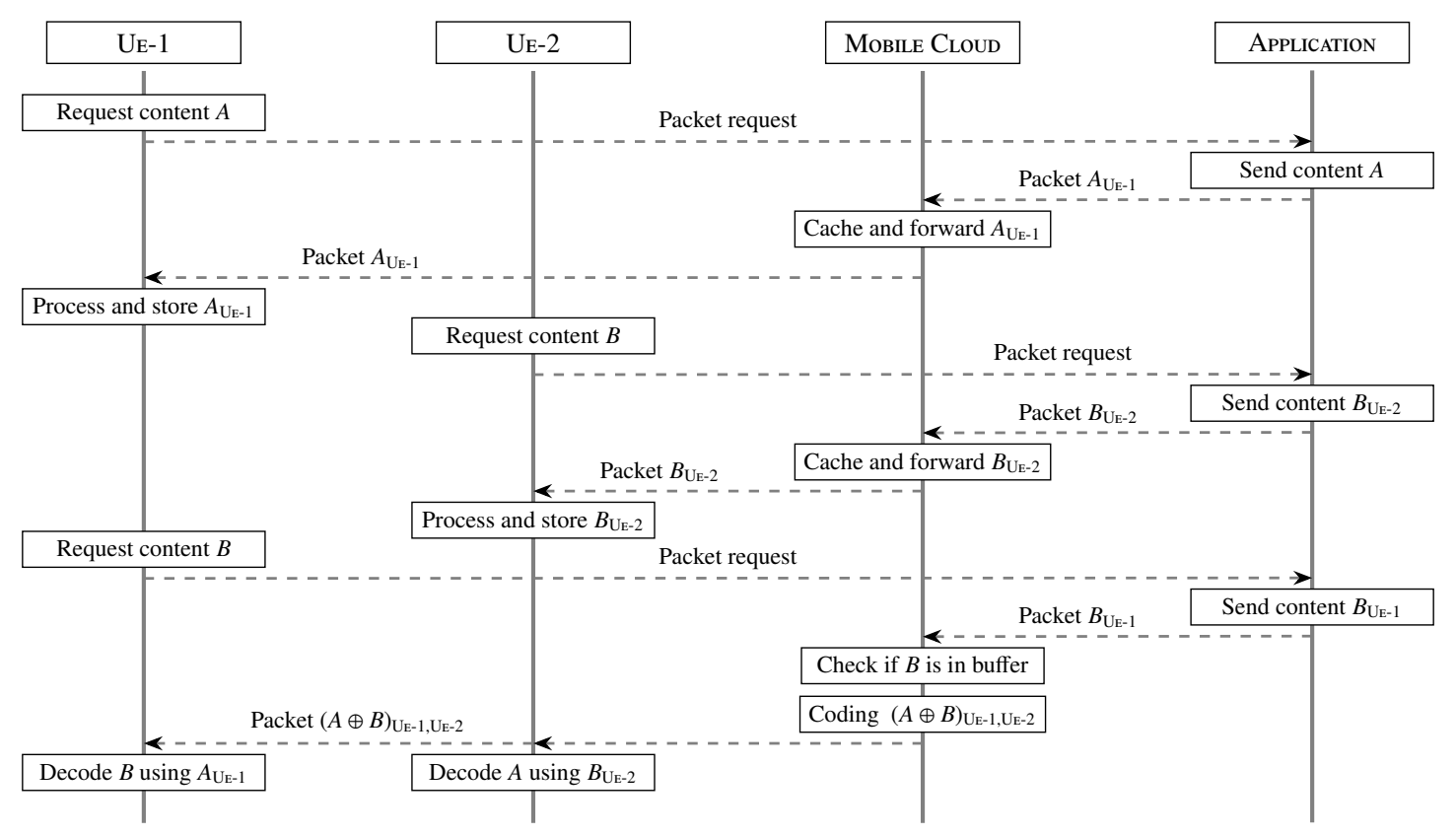

Fig. 1. vNC-CELL operation with two users

co-located users. NC-CELL allows cellular base stations to monitor in transit traffic and caching it locally. Then, whenever a coding opportunity is detected, which occurs when the base station can distribute information needed by two or more users with a single transmission, a coded packet is created and broadcast in the cell.

In this paper, we extend NC-CELL proposing virtualized NC-CELL (vNC-CELL). Finding coding opportunities and combining packets may become computationally expensive if executed locally at the base stations (eNodeBs). Having these operations virtualized and executed in a mobile cloud environment avoids additional computational overhead to eNodeBs.

The rest of the paper is organized as follows. Section II briefly illustrates the vNC-CELL technique used for content distribution and highlights the enhancements with respect to the previous proposal. Performance evaluation and results are presented in Section III. Finally, Section IV concludes the work outlining potential directions for future research on the topic.

\section{The VNC-CELL TeCHNiQue}

The proposed vNC-CELL approach is designed to optimize information delivery with partially overlapping content in cellular networks using network coding. vNC-CELL requires eNodeBs to monitor network traffic destined to the cloud users, cache it locally, and identify eventual coding opportunities. A coding opportunity occurs when eNodeB can distribute information with a single broadcast transmission that two or more users can use performing decoding.

Fig. 1 illustrates NC-CELL network coding performed for two users. First, users UE-1 and UE-2 request and obtain from the cloud content $A$ and content $B$ respectively. While content

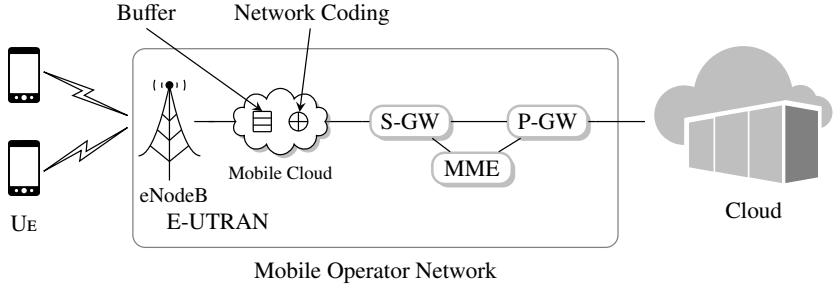

Fig. 2. System scenario

$A$ and content $B$ are passing through the cellular network, they are cached in a local buffer for possible encoding. Whenever any of the users request a content previously received by the other user (in this case user UE-1 requests content $B$, which was previously delivered to user UE-2) a coding opportunity arises. The eNodeB uses this opportunity to encode $A$ and $B$ and send them together in broadcast with a single coded packet $(A \oplus B)_{\mathrm{UE}-1, \mathrm{UE}-2}$. Having received $(A \oplus B)_{\mathrm{UE}-1, \mathrm{UE}-2}$, each user performs decoding by subtracting (applying XOR) previously received content, i.e., user UE-1 subtracts $A$, while user UE-2 subtracts $B$, for successful decoding.

Most of the aforementioned operations are computational intensive and may be not appropriate for the implementation in current eNodeBs. Therefore, in vNC-CELL network coding procedures are virtualized and executed in a mobile cloud (MC) environment similarly to [22]. Fig. 2 highlights separation of coding functionalities from eNodeBs. Decoupling computationally complex functions from eNodeBs is an emerging trend in cellular networks. Several works discuss integration of cloud computing and virtualization in cellular networks [8], [23], [24], [25] and foresee increased adoption of mobile clouds to better support multimedia services in cellular networks [22]. In 


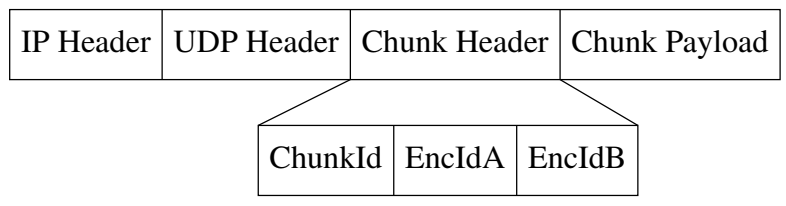

Fig. 3. Packet structure

the remainder of the paper by referring to eNodeBs we consider eNodeBs enhanced with coding functionalities executed in the MC. vNC-CELL operation for coding and decoding is similar to our previous proposal [12].

\section{Performance Evaluation}

\section{A. Evaluation Scenario}

For evaluating performance of vNC-CELL, the NS-3 network simulator, extended with LTE functionality from LENA project framework, was used. Fig. 2 illustrates simulation scenario A set of mobile users (UEs) is served by a single eNodeB connected to Packet data network GateWay (P-GW), which in turn is connected to the cloud. The interconnection links are point-to-point $10 \mathrm{~Gb} / \mathrm{s}$ links. We implemented vNC-CELL functionalities installing a MC environment attached to the eNodeBs as per Fig. 2.

Each mobile user requests a set of chunks independent of other users. The interval between two consecutive requests is uniformly distributed between 100 and $200 \mathrm{~ms}$. Random distribution of the requests makes the scenario more realistic than the that considered in numerical evaluation [12]. It becomes not possible to know the number of common chunks requested by the users a priori, which does not allow computing optimal allocation of coding opportunities. The simulation period is 10 seconds during which the cloud application delivers a set of 100 chunks. The chunks are delivered using 50 bytes long UDP protocol packets and have the structure presented in Fig. 3. Each chunk header consists of three 2-octet long fields, used for identification. ChunkId identifies the content sent with individual transmissions, while EncIdA and EncIdB identify encoded packets. For every chunk destined to mobile users, eNodeB stores chunk ID (ChunkId), destination address of the user and packet content in the buffer organized in the form of a hash table.

A coding opportunity arises when chunk ID of a packet about to be transited to a mobile user (primary user) has been already delivered to another user (secondary user). eNodeB encodes this in transit to the primary user chunk with another chunk which was already delivered to the primary user and broadcasts them together using a single transmission. The second chunk used for encoding is selected to maximize the number of users that would be able to decode both chunks after reception.

The process of chunk encoding is as follows. The payloads of two original packets are XORed to form a new payload. The original IDs are stored in the fields EncIdA and EncIdB of new chunk header. The ChunkId field is set to the predefined value " 0 ," which indicates an encoded chunk. For decoding, the users follow procedure illustrated in [12].

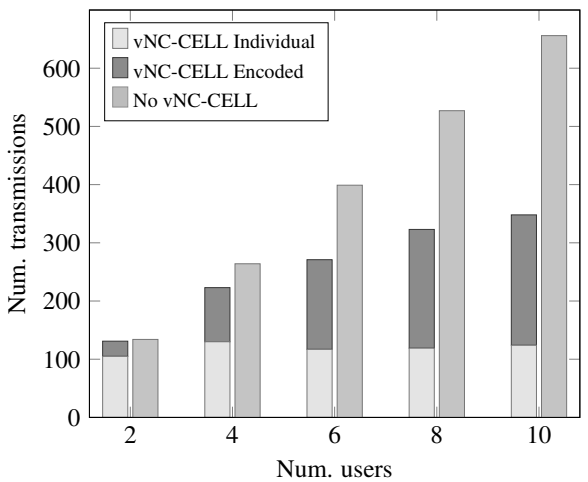

Fig. 4. Number of transmissions performed by eNodeB

\section{B. Results}

Fig. 4 shows the number of eNodeB transmissions. vNC-CELL always requires a smaller number of the transmissions and the difference becomes larger as the number of mobile users increases. Fig. 4 makes distinction between individual and encoded transmissions when vNC-CELL is enabled. Interestingly, the number of individual transmissions remains almost constant. It does not change with the increase in the number of mobile users active in the system. Instead, it correlates to the number of chunks the application can deliver. Once all or most of the chunks are buffered at eNodeB, every new in transit packet leads to a coding opportunity with a high probability. However, when eNodeB's buffer contains only a few chunks and just begins to fill up, the probability of having coding opportunities is low. As a consequence, eNodeB can not exploit completely network coding benefits and transmits non-encoded chunks more frequently.

The evolution of individual and encoded transmissions in time is depicted in Fig. 5. At the beginning of simulation, the number of individual transmissions increases rapidly and then, towards the end of the simulation, the increase becomes moderate (see Fig. 5(a)). On the contrary, the number of encoded transmissions shows an opposite behavior (see Fig. 5(b)). At the beginning, the eNodeB's buffer contains just a few packets and content is likely to be delivered using individual transmissions. However, as the buffer at eNodeB fills up, the probability of having coding opportunities increases and, as a result, the number of coded transmissions increases as well.

Having analyzed the vNC-CELL performance from the operator network perspective, we can now focus on the user side. Fig. 6 plots the number of content packets successfully received by the users and shows distribution of individual and encoded packets that were received. In total, each individual user receives more packets than it actually needs to decode. Therefore, the size of vNC-CELL bars is larger than the corresponding content-only bars. However, due to the fact that encoded packets are sent only once in broadcast, vNC-CELL can reduce the number of total transmissions if counted for all the users. As the number of users in the system increases, the 


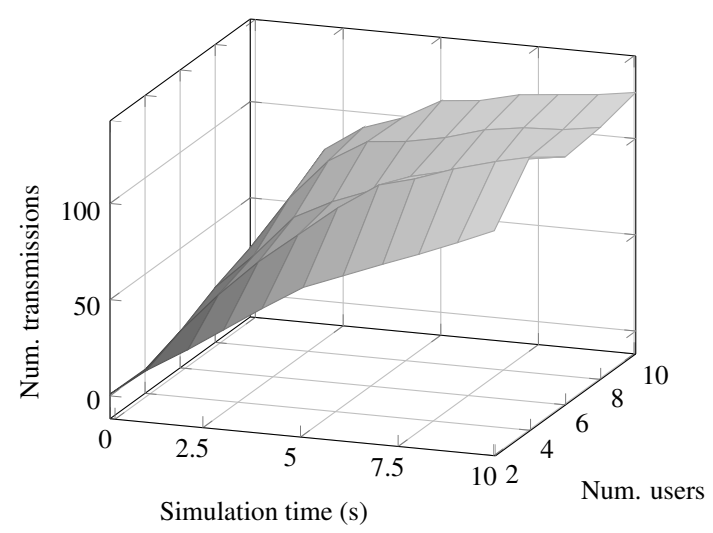

(a) Individual transmissions

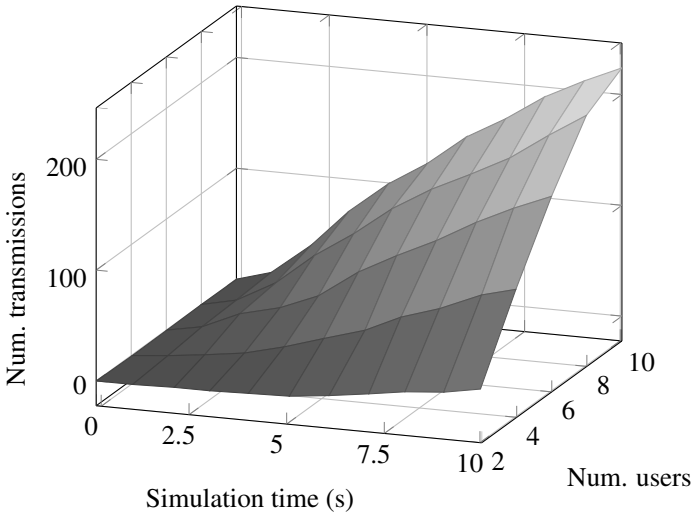

(b) Encoded transmissions

Fig. 5. Individual and encoded transmissions

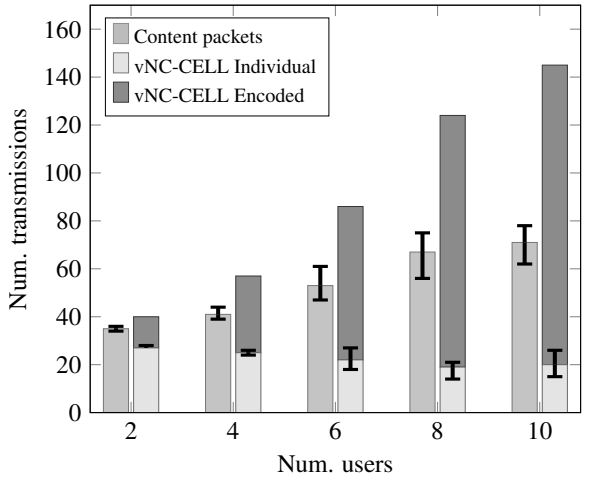

Fig. 6. vNC-CELL transmissions received by mobile users

number of individual transmissions decreases as most of the content begins to be distributed using encoded transmissions.

Having a large amount of encoded packets allows the users to retrieve content faster. This is a well known benefit of applying network coding for content distribution in P2P networks [16]. To highlight the details, Fig. 7 shows the time it takes to download a 10000 -chunk file by 4 users when each user is allowed to ask for only random 500 chunks. In this unfavorable situation, when only minor portion of chunks overlaps between users, vNC-CELL still achieves approximately $10 \%$ shorter download times.

The analysis provided so far considered an ideal channel without losses. However, in real world, path loss, multipath, fading and shadowing can cause packet losses and need to be taken into account. NS-3 simulator includes 11 different propagation loss models and two of them, COST-Hata and Log Distance Path Loss models, are the most suitable for urban and sub-urban scenarios [26]. For the simulations, the COST-Hata model was used.

Fig. 8 shows distribution of content, individual and encoded transmissions using vNC-CELLThe users are placed at an increasing distance from eNodeB in the range of $[50,500] \mathrm{m}$, being $500 \mathrm{~m}$ the typical range of pico cells in urban envi-

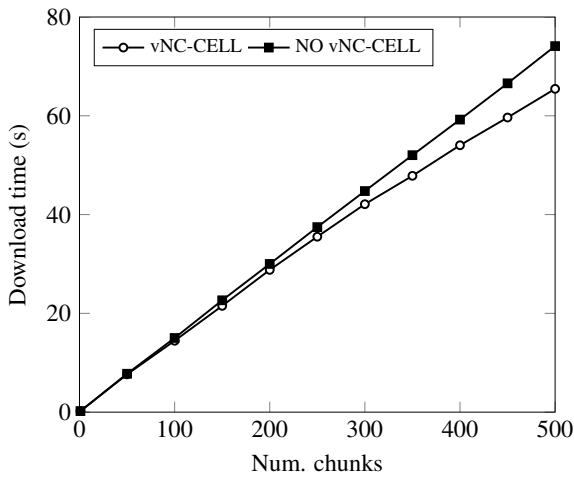

Fig. 7. Download time comparison

ronment [27]. As expected, after a certain threshold close to the cell border $(490 \mathrm{~m})$, the throughput performance reduces significantly. Fig. 8(b) underlines vNC-CELL scalability as the number of individual transmissions remains almost constant with the increase of the number of users in the system.

Fig. 9(a) analyses the download time and download time gain versus distance to mobile users. The latter is computed comparing performance of the system with and without vNC-CELL. For distances within $400 \mathrm{~m}$ the error rates are small and vNC-CELL performance improvement is stable around $10-15 \%$. While in the proximity of the cell border, between 450 and $490 \mathrm{~m}$, retransmission schemes start to fail and nodes lose their ability to cope with high error rates. The maximum gain is achieved in the proximity of $490 \mathrm{~m}$ and corresponds to $23.18 \%$ (see Fig. 9(b)). In the presence of high error rate, being not able to decode an encoded transmission is not an issue since the content carried by that packet can be retrieved from subsequent encoded transmissions. However, for distances beyond $490 \mathrm{~m}$ the loss rate increases dramatically and it becomes beneficial even not to enable vNC-CELL, as its performance improvement turns negative. 


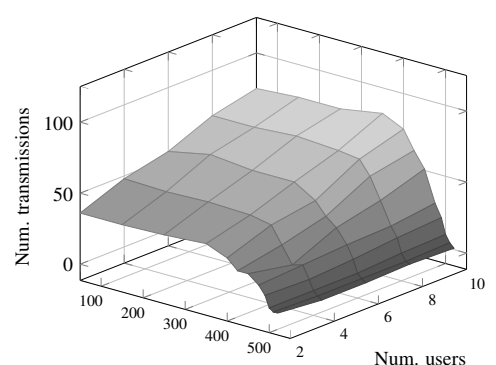

Distance from eNodeB (m)

(a) Content

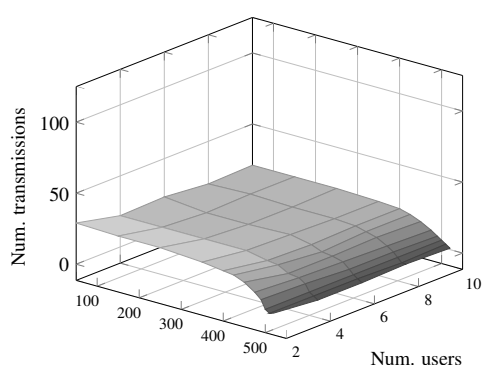

Distance from eNodeB (m)

(b) Individual

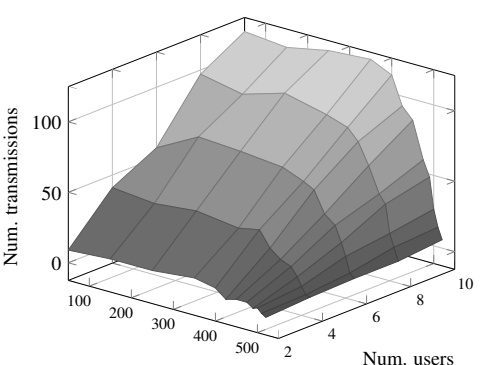

Distance from eNodeB $(m)$

(c) Encoded

Fig. 8. Distribution of content, individual and encoded transmissions in presence of channel errors

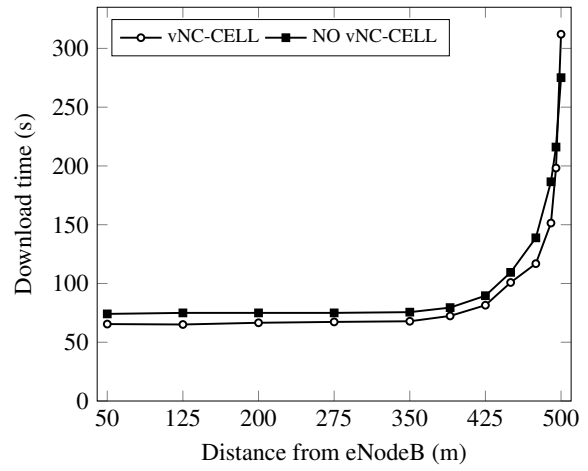

(a) Content download time

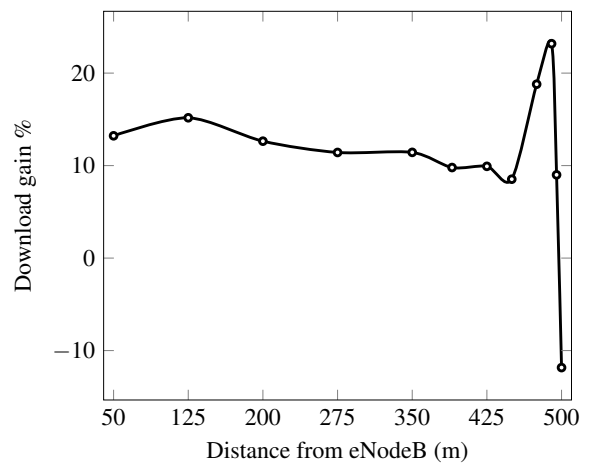

(b) Download time gain

Fig. 9. Analysis of download time with path losses

\section{CONCLUSION}

This paper presents vNC-CELL technique, which uses network coding for efficient content distribution in mobile cellular networks. With vNC-CELL eNodeBs monitor in transit traffic and optimize utilization of network resources combining flows with similar or partially overlapping content that has to be delivered to multiple users. Despite our previous proposal, vNC-CELL relieves base stations from computationally intensive coding operations virtualizing network coding functionalities in a mobile cloud environment.

The performance of the proposed technique has been validated through simulation experiments performed using NS-3 network simulator. Both user and operator perspective have been analyzed. The vNC-CELL approach is the most beneficial in systems with a large number of users and a large amount of the shared content. Besides improving network throughput, vNC-CELL allows users to retrieve content faster.

\section{ACKNOWLEDGMENT}

The authors would like to acknowledge the funding from National Research Fund, Luxembourg in the framework of ECO-CLOUD and iShOP projects.

\section{REFERENCES}

[1] "Global mobile statistics 2014," mobiThinking, 2014. [Online]. Available: http://mobithinking.com/mobile-marketing-tools/latest-mobile-stats
[2] H. Falaki, D. Lymberopoulos, R. Mahajan, S. Kandula, and D. Estrin, "A first look at traffic on smartphones," in Proceedings of the 10th ACM SIGCOMM Conference on Internet Measurement, ser. IMC ' 10. New York, NY, USA: ACM, 2010, pp. 281-287. [Online]. Available: http://doi.acm.org/10.1145/1879141.1879176

[3] H. T. Dinh, C. Lee, D. Niyato, and P. Wang, "A survey of mobile cloud computing: architecture, applications, and approaches," Wireless Communications and Mobile Computing, vol. 13, no. 18, pp. 1587-1611, 2013. [Online]. Available: http://dx.doi.org/10.1002/wcm.1203

[4] "Mobile cloud market," MarketandMarkets, 2014. [Online]. Available: http://www.marketsandmarkets.com/PressReleases/mobile-cloud.asp

[5] "Cisco visual networking index: Global mobile data traffic forecast update, 2014-2019," 2015, Cisco White Paper.

[6] A. Antonopoulos, E. Kartsakli, and C. Verikoukis, "Game theoretic d2d content dissemination in $4 \mathrm{~g}$ cellular networks," IEEE Communications Magazine, vol. 52, no. 6, pp. 125-132, June 2014.

[7] C. Ragona, C. Fiandrino, D. Kliazovich, F. Granelli, and P. Bouvry, "Energy-efficient computation offloading for wearable devices and smartphones in mobile cloud computing," in IEEE Global Communications Conference (GLOBECOM), Dec 2015.

[8] C. Fiandrino, D. Kliazovich, P. Bouvry, and A. Zomaya, "Networkassisted offloading for mobile cloud applications," in IEEE International Conference on Communications (ICC), June 2015, pp. 5833-5838.

[9] S. Andreev, A. Pyattaev, K. Johnsson, O. Galinina, and Y. Koucheryavy, "Cellular traffic offloading onto network-assisted device-to-device connections," IEEE Communications Magazine, vol. 52, no. 4, pp. 20-31, April 2014.

[10] I. Leontiadis, C. Efstratiou, M. Picone, and C. Mascolo, "Don't kill my ads!: Balancing privacy in an ad-supported mobile application market," in Proceedings of the Twelfth Workshop on Mobile Computing Systems \& Applications, ser. HotMobile '12. New York, NY, USA: ACM, 2012, pp. 2:1-2:6. [Online]. Available: http://doi.acm.org/10.1145/2162081.2162084

[11] N. Vallina-Rodriguez, J. Shah, A. Finamore, Y. Grunenberger, 
K. Papagiannaki, H. Haddadi, and J. Crowcroft, "Breaking for commercials: Characterizing mobile advertising," in Proceedings of the 2012 ACM Conference on Internet Measurement Conference, ser. IMC '12. New York, NY, USA: ACM, 2012, pp. 343-356. [Online]. Available: http://doi.acm.org/10.1145/2398776.2398812

[12] C. Fiandrino, D. Kliazovich, P. Bouvry, and A. Zomaya, "NC-CELL: Network coding-based content distribution in cellular networks for cloud applications," in IEEE Global Communications Conference (GLOBECOM), Dec 2014, pp. 1205-1210.

[13] R. Ahlswede, N. Cai, S.-Y. Li, and R. W. Yeung, "Network information flow," IEEE Transactions on Information Theory, vol. 46, no. 4, pp. 1204-1216, 2000.

[14] S.-Y. Li, R. W. Yeung, and N. Cai, "Linear network coding," IEEE Transactions on Information Theory, vol. 49, no. 2, pp. 371-381, 2003

[15] T. Matsuda, T. Noguchi, and T. Takine, "Survey of network coding and its applications." IEICE Transactions, vol. 94-B, no. 3, pp. 698-717, 2011.

[16] C. Feng and B. Li, "Network coding for content distribution and multimedia streaming in peer-to-peer networks," Network Coding: Fundamentals and Applications, pp. 61-86, 2012.

[17] A. Antonopoulos and C. Verikoukis, "Multi-player game theoretic mac strategies for energy efficient data dissemination," IEEE Transactions on Wireless Communications, vol. 13, no. 2, pp. 592-603, February 2014.

[18] Z. Lv, K. Xu, and Y. Xu, "A practical HARQ scheme with network coding for LTE-A broadcasting system," International Conference on Wireless Communications Signal Processing (WCSP), pp. 1-6, 2012.

[19] J. Li, B. Jia, Z. Hu, and Y. Wang, "Design of joint network and channel coded relay in LTE-A system," IEEE 13th International Conference on Communication Technology (ICCT), pp. 1124-1129, Sept 2011.
[20] Y. Lang, D. Wubben, A. Dekorsy, V. Braun, and U. Doetsch, "Improved HARQ based on network coding and its application in LTE," IEEE Wireless Communications and Networking Conference (WCNC), pp. 19581963, 2012.

[21] D. Vukobratovic, C. Khirallah, V. Stankovic, and J. Thompson, "Random network coding for multimedia delivery services in LTE/LTE-Advanced,' IEEE Transactions on Multimedia, vol. 16, no. 1, pp. 277-282, 2014.

[22] M. Felemban, S. Basalamah, and A. Ghafoor, "A distributed cloud architecture for mobile multimedia services," IEEE Network, vol. 27, no. 5, pp. 20-27, September 2013.

[23] Y. Cai, F. Yu, and S. Bu, "Cloud computing meets mobile wireless communications in next generation cellular networks," IEEE Network, vol. 28, no. 6, pp. 54-59, Nov 2014.

[24] J. Liu, T. Zhao, S. Zhou, Y. Cheng, and Z. Niu, "CONCERT: a cloudbased architecture for next-generation cellular systems," IEEE Wireless Communications, vol. 21, no. 6, pp. 14-22, December 2014.

[25] G. Karagiannis, A. Jamakovic, A. Edmonds, C. Parada, T. Metsch, D. Pichon, M. Corici, S. Ruffino, A. Gomes, P. Secondo Crosta, and T. Bohnert, "Mobile cloud networking: Virtualisation of cellular networks,' 21st International Conference on Telecommunications (ICT), pp. 410-415, May 2014.

[26] M. Stoffers and G. Riley, "Comparing the NS-3 propagation models," IEEE 20th International Symposium on Modeling, Analysis \& Simulation of Computer and Telecommunication Systems (MASCOTS), pp. 61-67, 2012.

[27] R. K. Jain, S. Katiyar, and N. K. Agrawal, "Hierarchical cellular structures in high-capacity cellular communication systems," CoRR http://arxiv.org/abs/1110.2627, 2011. 